\title{
REFERENNCIA
}

\section{CONTRIBUICCĀO AO ESTUDO DOS ELEMENTOS VASCULARES ARTERIAIS E VENOSOS, HILARES E EXTRAHILARES, EM RINS DE CAPRINOS DA RAÇA MOXOTO}

MARIA ALELINA MARTINS DE CARUALHO

Professora Adjunta Universidade Federal do Piaul

JOAO GILBERTO LOPES PEREIRA Professor Adjunto Faculaade ae Medicina Veterinaria e Zootecnia da USP

CARURLHO, M.A.M. \& PEREIRA, J.G.L. Contribuicaro ao estudo dos elementos vasculares arteriais e venosos, hilares e extrahilares, em rins de capinos da raca Moxoto. Rev. Fac. Med. Vet. Zootec. Univ. S. Paulo, 24(2):119-126, 1987.

RESUMO: 0 trabalho baseia-se no exame de 30 conjuntos, abrangendo cada um rins, vasos, ureteres e tractos correspondentes da arteria arta e veia cava caudal, retirados de caprinos a raca Moxato, machos, jovens - adultos, oriundos do estado de Pernambuco, nos quais se estudaram o numero os locais de entrada e salda dos ramose ralzes hilares e extranilares, respectivamente das arterias e veias renais. O material assim ootido ioi rixaco en soluczo aquara de formol a 10\%, sendo, a seguir, dissecado com aux!lio de lupa.

UNITERMOS: Anatomia, caprinos: Rim; Arte$r i a s ; V e i a s$

\section{INTRODUCAO E LITERATURA}

- caprino da raca Moxoto representa animal de grande valor zootecnico na regiao Nordeste do Brasil, merecendo, desta maneira, a atencão dos pesquisadores no sentido de fornecer substios que venham colaborar para melhor conhecimento e consequente aprimoramento des. ta raca.

Nesta oportunidade, seguindo o mesmo procedimenta que outros autores ja utilizaram dentro da linha de pesquisa em desenvolvimento nas Disciplinas de Anatomia do Departamento de Cirurgia e Dbstetrleia da Faculdade de Medicina Veterinaria e Zootecria da Universidade de Sao Paulo, propomo-nos a estudar a disposicão dos elementos vasculares renais nesta raca de caprinos, analisando aspectos relativos jo numero e as regires de entrada e salda, respectivamente, dos ramos arteriais e ralzes venosas dos rins destes especimes, com a finalidade de obter informacőes sobre essas estruturas, visando, assim melhor enfoque dos aspectos anatomicas da citada raca e contriouindo para o desenvolvimento da Anatomia Lomparativa.

\section{MATERIAL E METODO}

Para a concretizacão desta pesquisa, examinamos 30 pares de rins, recolhidos de caprinos da raca Moxoto, machos, jovens e adultos, oriundos de varias regiales de eriacăo do estado de Pernambuco, cedidos pelo Centro Nacional de Pesquisas de Caprinos.

Os referidos orgãos, retirados convenientemente da cavidade aboominal, mantidos aos pares, unidos por suas arterias e veias aos tractos respectivamente, da arteria aorta e veia cava caudal, foram dissecados sob lupa circuLar de luz fria "Referència LL 20", após fixaço em soluço aquosa de formol a 10,0\%, durante pelo menos 48 horas, evidenciando, desta forma, os componentes vasculares das regides hilar e extrahilar.

Para o relato dos resultados, consideramos as regizes em apreco, demarcando nelas os quadrantes cranioventral. craniodorsal, caudoventral e caudodorsai mediante cuas linnas imaginarias: uma longitudinal, tracada de oblo a polo do 
rim, representando.o ciametro craniocaudab, e a outra equivalente ao diametro oorsoventral, perpendicular à primeira, interceptando-a exatamente no centro da seccáo transversa justarenal do ureter. Por fim, registramos em desenhos esquemaicicos que coniguram os pares de rins subinetioos a um giro de $90^{\circ}$, no sentido medioventral, tanto o número como os locais de entrada e salja dos ramos arteriais e das ralzes venosas, levanoo-se em conta nao apenas os diferentes quadrantes, mas as regioes neles assinaladas.

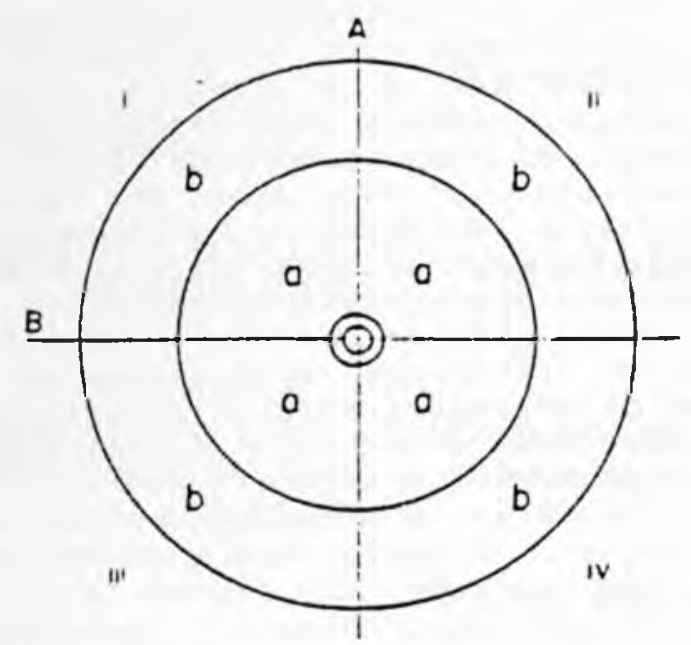

D

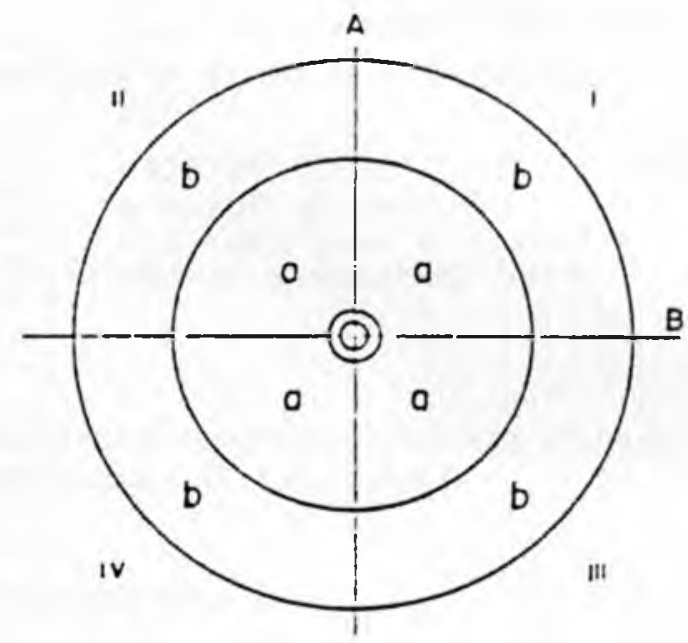

$\mathbf{E}$

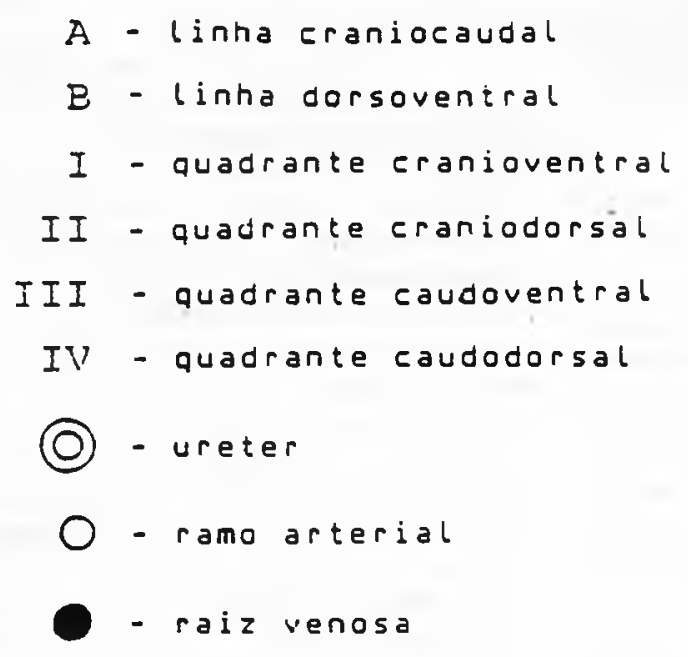

a - regiazo hilar

b - regiao extrahilar

d - rim direito

e - rim esquerdo 
A analise estatistica dos dados obtidos foi realizada graca a utilizacáo do coeficiente de correlacro de Pearson ao nivel de $5,0 \%$.

\section{RESULTADOS}

Para os 80 orgaros estudados, expomos os dados correspondentes ao numero, disposicaro e regiles de entrada e salda, respectivamente, dos ramos da arteria renal e das ralzes da veia renal, obedecando os quacrantes e linhas previamente tracados para os rins direito e esquerdo, atraves dos quadros $1,2,3$ e 4 .

\section{COMENTARIOS}

Conforme esclarecemos anteriormente quanto às informaçles atinentes aos elementós vasculares renais em caprinos, nada encontramos nos livros de texto consagrados a Anatomia Veterinaria. A maioria dos tratadistas, na realidade, toma o gauino como padrao em suas deserifr̆es, sem contudo adontar, em relaça ao focado assunto, as posslveis diferencas existentes para os caprinos quando considera comparativemante a Anatomia dos Animais Jomésticos. A esse respeito, cumpre-nos ressaltar que, mesmo-aqueles autores que se preocupam especialmente com os ruminantes, como MONTANE \& JOURDELLE, 3 e MARTIN \& SCHAUDER, 2 , na o of erecem em seus comoendios dados pertinentes as variacães anatamicas relativas aos aludioos elementos vasculares em caprinos, capazes de permitirem confronlo com os resultados agora obtidos. Par outro lado, no tocante aos trabalhos especializados, deter-nos-emos, com naior cuidado, a cobejar nossos resultaJos com os de GUARENTI 3 FERNANDES FILHO, 1, Dois referem-se especilicamenle a caprinos, ainda que sem raca definida. Também, na medida do positivel, estabeleceremos conironto com os achados de PEDUTI NETJ, 4. emoora em ovinos da -aca Merino, por tratar-se de informa= đes concernentes a pequenos ruminantes. -embraremos, ainda., o fato de terem estes auiores utilizado a nesina netodologia aplicada em nossa pesquisa.

Assim, no referente do número de ramos arteriais, GUARENTI 3 FERNANDES
FILHO, 1, verificaram, junto ao hilo, a presenca de 5 a 14 ramos no rim direito e de 8 a 18 no esquerdo. Jà PEDUTI NETO, 4 registrou de 3 a 14 e 4 a 13 para 05 rins direito e esquerdo, respectivamente, enquanto em nosso trabalho consignamos valores que muitos deles se aproximam, vale dizer, de 7 a 13 a direita e de 6 a 12 a esquerda.

No que tange às raizes da veia renal, vistas na regiao hilar, nossos resultados apontam para os caprinos da raca Moxoto, de 2 a 7 e de 2 a 9 , respectivamente, a direita e esquerda, arranjo este observado por GUARENTI \& FERNANDES FILHO, 1, com numeros quase iguais, isto e, de 3 a 8 e de 3 a 7 obedecendo a mesma ordem de citacão, registro muito proximo do verificado por PEDUTI NETO, 4, ou seja, 2 a 7 tanto à direita como a esquerda.

Desejamos, ainda, destacar que o numero de ramos arteriais de posicao extranilar foi registrado por GUARENTI \& FERNANDES FILHO, 1 , variando de 1 a 3 para o rim direito, e de 1 a 4 para o esquerdo, valores estes que coincidem com os assinalados por nos.

Quanto ao numero de ralzes venosas a emergirem da citada regizo, GUARENTI \& FERNANDES FILHO, 1 , verificaram a mesma variaça numerica apontada para os ramos arteriais, ou seja, de 1 a 3 , e de 1 a 4. para os rins direito e esquerdo, respectivamente, enquanto em nossas pecas encontramos, apenas 1 vez, a esquerda, uma raiz venosa de localizacao extrahilar.

No tocante a disposica dos elementos vasculares renais, nosso estudo revelou que, tanto os ramos arteriais, como as ralzes venosas, acham-se em localizacao prevalentemente periferica em relacăo a regiao hilar dos rins direito e esquerdo, aspecto semelhante visto na pesquisa de GUARENTI \& FERNANDES FILHO, 1 e tambem registrado na maioria dos casos estudados por PEDUTI NETO, 4.

De outra parte, JUARENTI \& FERNANDES FiLHO, 1, examinando o povoamento dos quadrantes pelos ramos arteriais de ambos os rins, comoutaram maior concentracro destes vasos no quadrante cranioventral, fato este ocservado tambem em nossa investigaço. Ja para as ralzes venosas aqueles autores registraram maior contingente vascular no quadrante cranioventral, enauanto os nossos achados o revelam no quadrante caudoventral. Analisando o numero de ramos arteriais dos rins direito e esquerdo do mesmo animal, consignamos coincitêneia 
numerica 7 vezes $(23,3 \%)$, dado este que muito se aproxima daqueies observados por GUARENTI \& FERNANDES FILHO, 1 e PEDUTI NETO, 4, isto e, pela ordem, 4 vezes $(13,3 \%)$ e 5 vezes $(16,7 \%)$, enquanto no referente as ralzes venosas, registramos tal comportamento em 8 vezes $(26,7 \%)$, resultado este que coincide com - de GUARENT! \& FERNANDES FILHO, 1; entretanto, os achados de PEDUTI NETO, 4. apresentaram frequencia bem acima da verificada pcr nos, vale dizer, 15 vezes $(50,0 \%)$.

Por rim, analisando estatisticamente os nossos dados, verificamos correlaça positiva nao significante ao nivel de 5,0\% entre a numero de ramos arteriais e ralzes venosas situados no nilo $0_{7}$ seja para o rim direito, seja para - rim esquerdo, valor este concordante com os assinalados por GUARENTI \& FERNANDES FILHO, $\uparrow$.

\section{CONCLUSOES}

Os resultados ootidos mediante o exame de 60 rins de caprinos da raca Moxoto, machos, jovens e adultos, permitiram-nos chegar as seguintes concluş̧еs:

1. Os ramos da arteria renal computajos na regiăo hilar são vistos no rim direito em numero de oito $(33,3 \%)$, nove $(20,0 \%)$, dez $(16,7 \%)$, onze $(13,3 \%)$, se:e $(13,3 \%)$, treze $(3,3 \%)$, e, no esquerdo, nove $(33,3 \%)$, dez $(23,3 \%)$, oito $(13,3 \%)$, doze $(10,0 \%)$, onze $(10,0 \%)$, sete $(6,7 \%)$, seis $(3,3 \%)$.

2. Os ramos arteriais consignados na zona extrahilar do rim direito săo identificados em numero de um $(13,3 \%)$, três $(6,7 \%)$, dois $(6,7 \%)$, e, no esquerdo, um $(16,7 \%)$, quatro $(3,3 \%)$, très $(3,3 \%)$.

3. No que tange as ralzes venosas, junto ao hilo, no rim direito, contam-se quatro $(36,7 \%)$, três $(33,3 \%)$, cinco $(13,3 \%)$, sete $(6,7 \%)$ seis $(6,7 \%)$, duas $(3,3 \%), e$, no esquerdo, cinco $(33,3 \%)$, trés $(30,0 \%)$, quatro $(20,0 \%)$, seis $(6,7 \%)$, nove $(3,3 \%)$, sete $(3,3 \%)$, duas $(3,3 \%)$.
4. No atinennte às palzes venosas na regiao extranilar, verifica-se apenas uma $(3,3 x)$, à esquerca.

5. Os ramos da arteria renal, bem como as ralzes venosas oos rins direito e esquerdo, acham-se na regiao hilar, em posicão preoominantemente periferica.

6. Nos 30 pares de rins dissecados, registramos, para os rins direito e esquerdo, igual numero de ramos arteriais, 7 vezes $(23,3 \%)$, disposiczo esta observada $g$ vezes $(25,7 \%)$ em relaça as ralzes venosas.

7. 0 maior povoamento vascular arterial, para amoos os rins, ocorre no quadrante cianioventral, enquanto que as ralzes venosas ocupam, com maior frequencia, o quadrante caudoventral.

8. A analise estatlstica (coeficiente de correlacăo.de Pearson) demonstra correlaça positiva nao significante ao nlvel de $5,0 \%$ entre o numero de ramos arteriais e ralzes venosas localizados no nilo, seja para o rim direito, seja para o esquerdo.

CARUALHO, M.A.M. \& PEREIRA, J.G.L. Contribution to the study of the vascular, arterial and venous elements hilar and extranilar - in kianeys of Moxotó caprines. Rev. Fac. Med. Vet. Zootec. Univ. 5. Paulo, 24(2):119-126, 1987.

SUMMRRY: inis work is based on the examination of 30 animals, whose kidneys, vessels. ureters and tracts related to the aorta artery ano caudal vena cava, extracted from young and adult, male Moxoto caprines from the state of Pernambuco, had the number and sites of penetration and outlet of the hilar ano extranilar roots and branches, respectively of the renal arteries and vessels, studied. The material thus obtained was fixed in aqueous solution of formol at 10.0\%, then dissected with the help of a magnifying glass.

UNITERMS: Anatomy of caprine; Kidney; Arteries; Veins 
Contribuição ao estudo dos ela:nentos vasculares, arteriais e venosos, hilares e extrahilares, em rins de caprinos da raça Moxotó
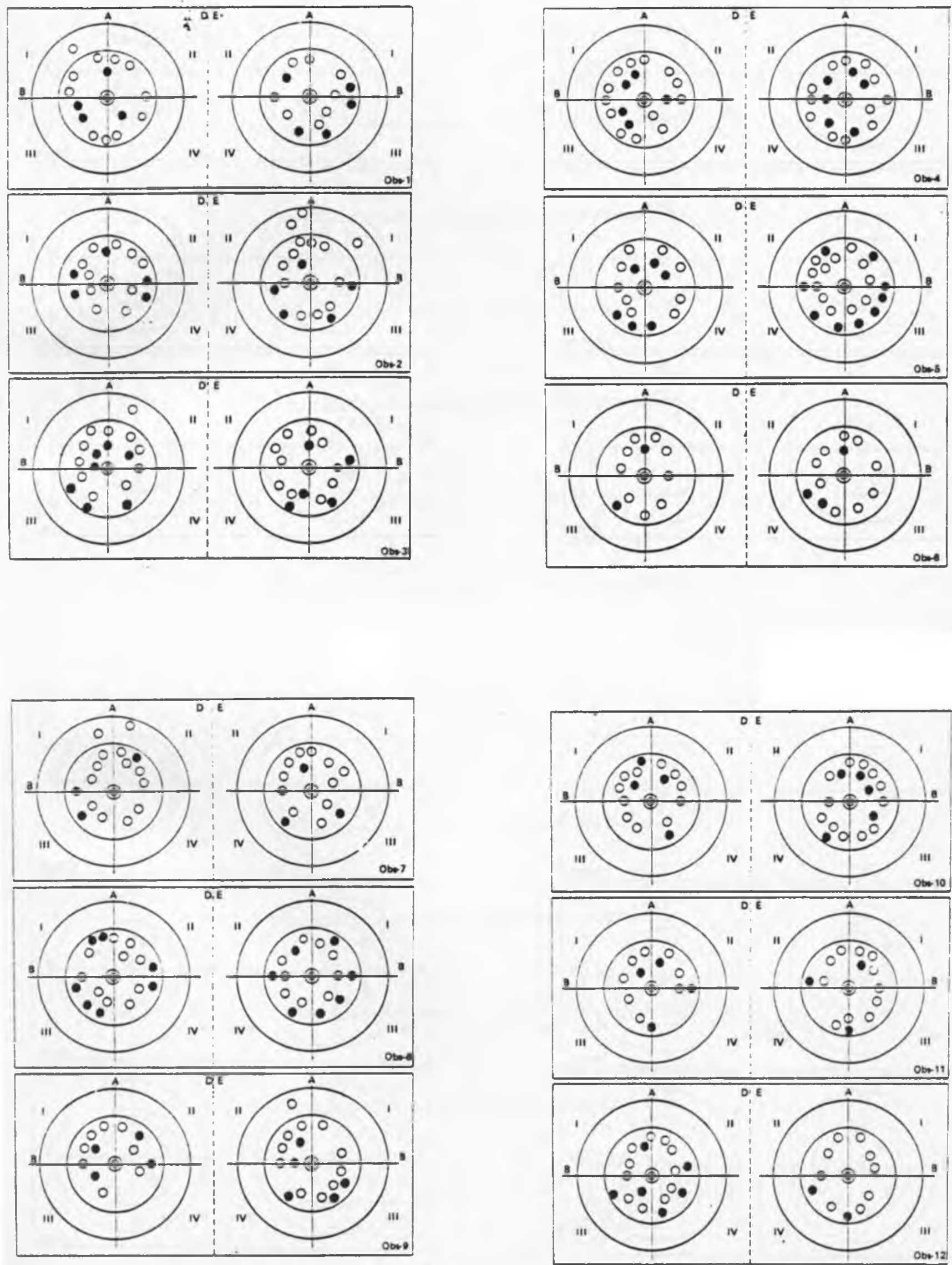

Rev.Fac.Hed.Ver.Zoorec.Univ.S.Paulo, 24(2):119-126, 1987. 

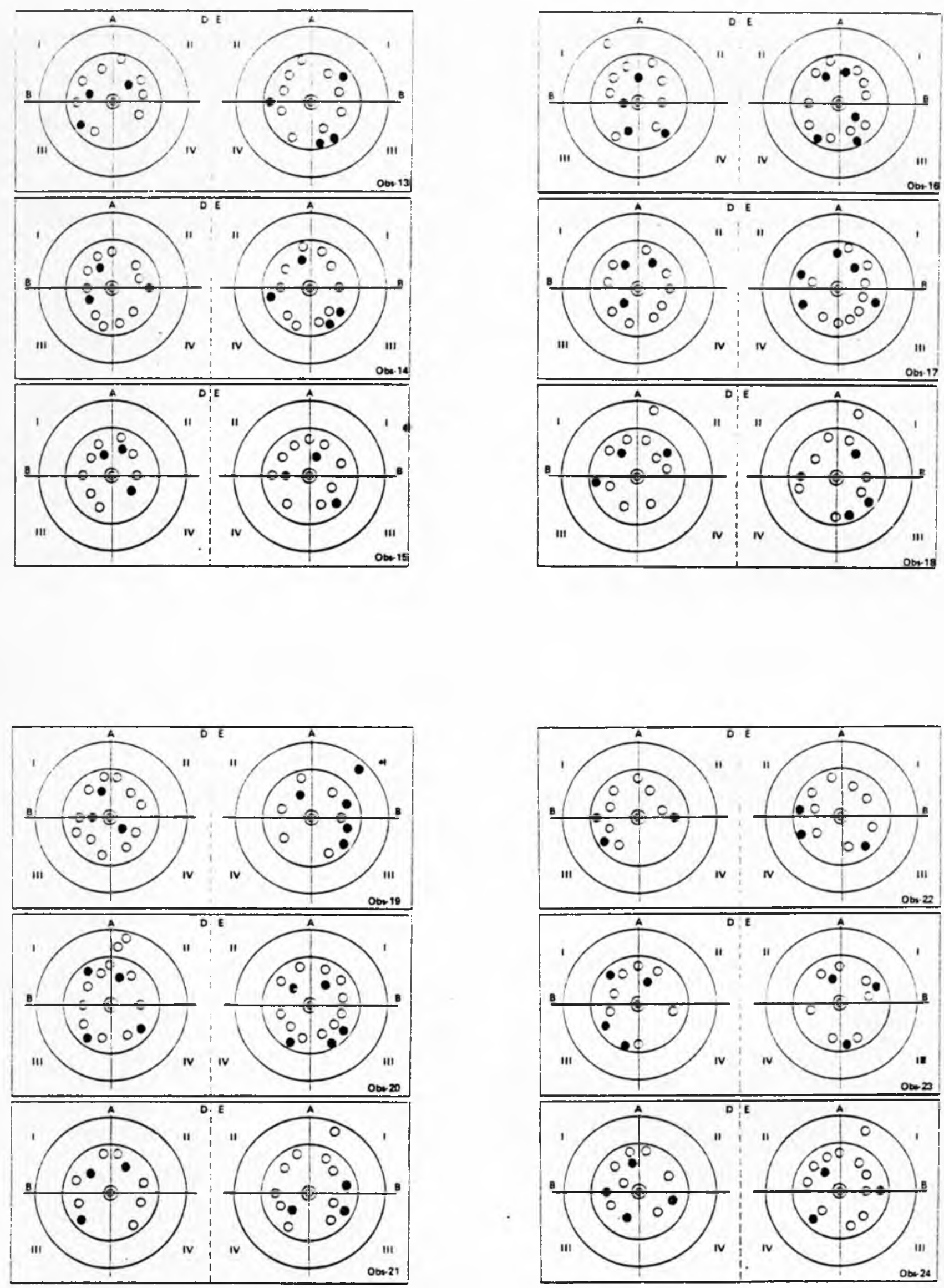

Rev.Fac.Med.Vet.Zootec.Univ.S.Paulo, 24(2):119-126, 1987. 

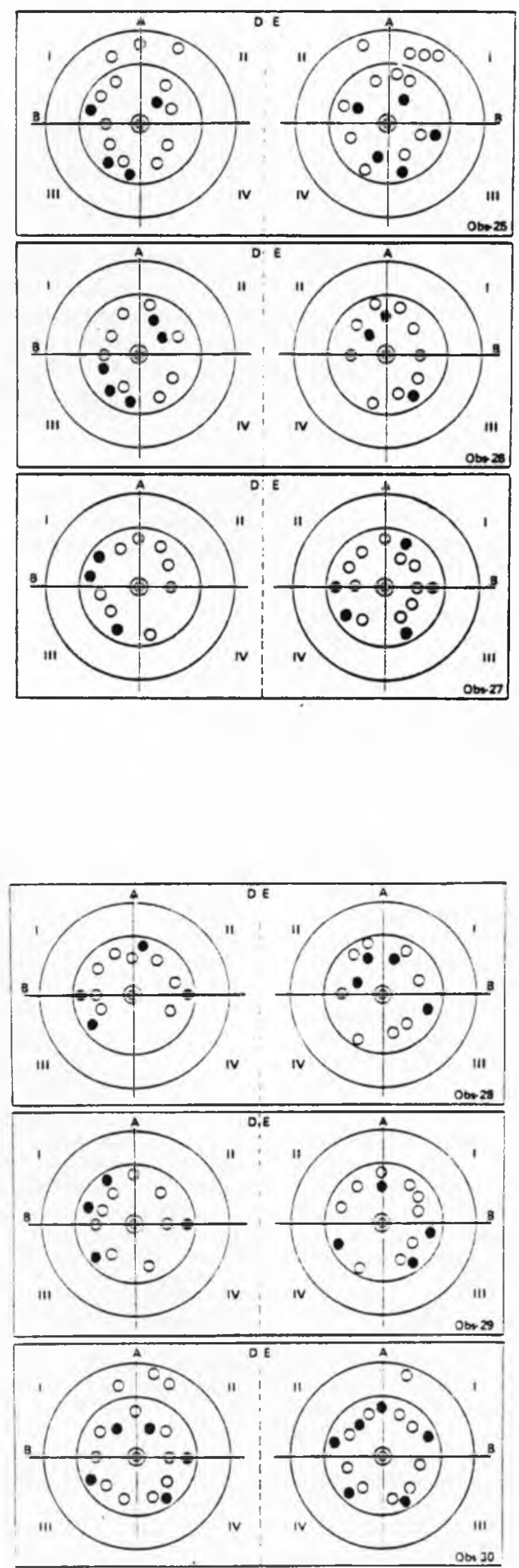

Rev.Fac.Med.Ver.Zootec. Univ.S.Parlo, 24(2):119-126, 1987. 
126 CARVALHO, M.A.M. \& PEREIRA, J.G.L.

REFERENCIAS BIBLIOGRAFICAS

1 - GUARENTI, V.P.J. \& FERNANDES FILHO, A. Contribuicáo ao estudo dos ramos arteriais e das raizes venosas, hilares e extrahilares, em rins de caprinos (Capra hir(us). Rev. Fac. Med. Vet. Zooter. Univ. 5. Paulo, 22:115193, 1985 .

2 - MARTIN, P. \& SEHAUDER, $w$. Lehrbuch der Anatomie der Haustiere. 5tuttgart, Jcnickhardt \& Ebner. 1938. v.3. p.277-278.
3 - MONTANE, L. \& BOURDELLE, E. Anatomie regionale des animaux domestiques. Paris, J.B.Baillière, 1917. v.2, p.300-302.

4 - PEDUTI NETO, J. Contribuicá ao estudo dos elementos vasculares, arteriais e venosos da hilo renal em ovinos (Ovis aries. Linnaeus, 1758). Rev. Fac. Med. Vet. Zootec. Univ. 5. Paulo, $13: 57-83,1976$.

Recebido para publicacaro em 15/04/87 Aprovado para publicafão em 03/07/87 